\title{
Identification of a missense $H O X D 13$ mutation in a Chinese family with syndactyly type I-c using exome sequencing
}

\author{
HAO DENG ${ }^{1,2}$, TING TAN ${ }^{1}$, QUANYONG HE ${ }^{3}$, QIONGFEN LIN ${ }^{4}$, ZHIJIAN YANG ${ }^{1}$, \\ ANDING ZHU ${ }^{2}$, LIPING GUAN ${ }^{4}$, JINGJING XIAO $^{4}$, ZHI SONG $^{2}$ and YI GUO $^{1,5}$ \\ ${ }^{1}$ Center for Experimental Medicine; Departments of ${ }^{2}$ Neurology and ${ }^{3}$ Burn and Plastic Surgery, \\ The Third Xiangya Hospital, Central South University, Changsha, Hunan 410013; ${ }^{4}$ BGI-Shenzhen, \\ Shenzhen, Guangdong 518083; ${ }^{5}$ Information Security and Big Data Institute, \\ Central South University, Changsha, Hunan 410083, P.R. China
}

Received January 7, 2016; Accepted February 2, 2017

DOI: $10.3892 / \mathrm{mmr} .2017 .6576$

\begin{abstract}
Syndactyly is one of the most common hereditary limb malformations, and is characterized by the fusion of specific fingers and/or toes. Syndactyly type I-c is associated with bilateral cutaneous or bony webbing of the third and fourth fingers and occasionally of the third to fifth fingers, with normal feet. The aim of the present study was to identify the genetic basis of syndactyly type I-c in four generations of a Chinese Han family by exome sequencing. Exome sequencing was conducted in the proband of the family, followed by direct sequencing of other family members of the same ancestry, as well as 100 ethnically-matched, unrelated normal controls. A missense mutation, c.917G $>$ A (p.R306Q), was identified in the homeobox D13 gene (HOXD13). Sanger sequencing verified the presence of this mutation in all of the affected family members. By contrast, this mutation was absent in the unaffected family members and the 100 ethnically-matched normal controls. The results suggest that the c.917G $>$ A (p.R306Q) mutation in the HOXD13 gene, may be responsible for syndactyly type I-c in this family. Exome sequencing may therefore be a powerful tool for identifying mutations associated with syndactyly, which is a disorder with high genetic and clinical heterogeneity. The results provide novel insights into the etiology and diagnosis of syndactyly, and may influence genetic counseling and the clinical management of the disease.
\end{abstract}

\section{Introduction}

Syndactyly is one of the most common congenital limb malformations and is associated with webbing of adjacent fingers/toes (1). It is a heterogeneous hereditary disease, with

Correspondence to: Professor Yi Guo, Information Security and Big Data Institute, Central South University, 932 South Lushan, Changsha, Hunan 410083, P.R. China

E-mail: yiguo0816@yahoo.com

Key words: syndactyly, homeobox D13 gene, p.R306Q, missense mutation, exome sequencing a prevalence of between 3 and 10 in every 10,000 births; however, a higher prevalence of between 10 and 40 in every 10,000 births has been reported $(1,2)$. In addition, it is twice as common in males, indicating that this disease may demonstrate incomplete penetrance (3). Syndactyly exists either as an isolated non-syndromic anomaly or as part of $>300$ syndromic abnormalities. Non-syndromic syndactyly may be cutaneous or bony and unilateral or bilateral (4). To date, at least nine types (I-IX) of phenotypically diverse non-syndromic syndactyly have been identified. Five primary types (type I-V) are inherited as an autosomal dominant trait with variable disease phenotypes and incomplete penetrance (4); while the remaining types and subtypes may demonstrate autosomal recessive or X-linked hereditary patterns of inheritance (3). The phenotype of a specific patient appears to be dependent on a specific gene, as well as their genetic background and the subsequent signaling pathways involved in limb formation (3). Non-syndromic syndactyly is genetic in origin (5). At present, at least 11 disease loci and eight disease-associated genes have been identified, including the homeobox D13 gene (HOXD13), the fibulin 1 gene, the gap junction protein alpha 1 gene, the limb development membrane protein 1 gene, the LDL receptor related protein 4 gene, the gremlin 1 gene, the formin 1 gene and the fibroblast growth factor 16 gene $(1,6)$. Mutations in the HOXD13 gene are responsible for the development of syndactyly type II-a and syndactyly type V $(7,8)$. The typical clinical features of syndactyly type I-c include bilateral cutaneous or bony fusion of the third and fourth fingers and occasionally of the third to fifth fingers, with normal feet (2).

The aim of the present study was to identify the genetic basis of syndactyly type I-c in four generations of a single Chinese family.

\section{Materials and methods}

Participators and clinical data. The present study enrolled a single Chinese Han family (15 members), and clinical data from physical examinations and peripheral blood samples were collected from 11 members (generation:family member: II:1, male, 64 years old; II:3, male, 54 years old; II:4, female, 51 years old; III:1, female, 41 years old; III:2, female, 39 years 
A

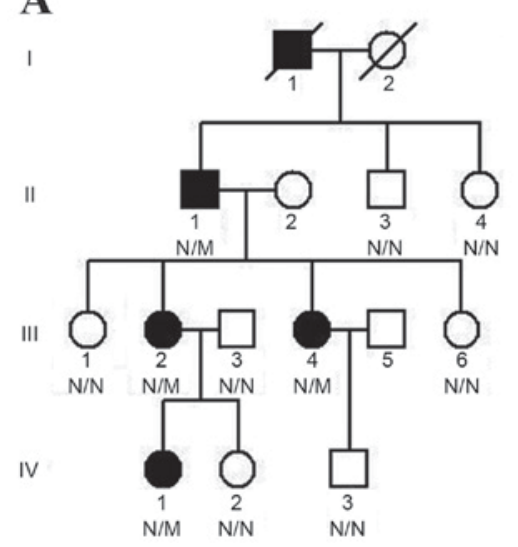

B

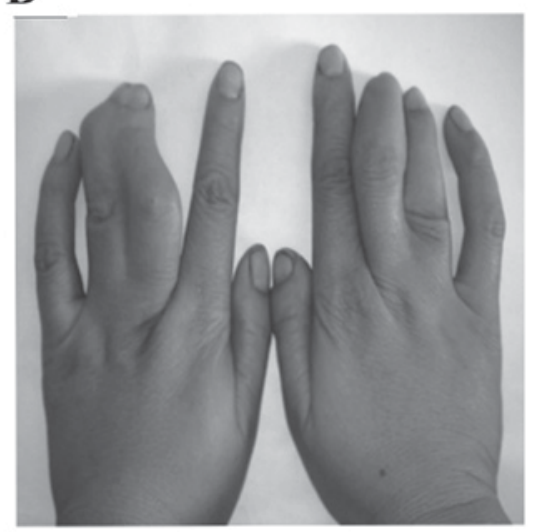

Figure 1. (A) Ancestry of the Chinese family with syndactyly type I-c. Squares and circles represent males and females, respectively; black squares and circles represent affected individuals; a diagonal slash through the symbol indicates that the individual was deceased at the time of the study. (B) Representative image of the phenotypic appearance of an affected member (generation III, member 2) in the family. N, normal allele; M, HOXD13 c.917G>A (p.R306Q) mutant allele; HOXD13, homeobox D13 gene.

old; III:3, male, 39 years old; III:4, female, 38 years old; III:6, female, 35 years old; IV:1, female, 16 years old; IV:2, female, 8 years old; IV:3, male, 15 years old) of the four-generation family enrolled at the Third Xiangya Hospital of Central South University in April 2013 (Changsha, China; Fig. 1A); 2 family members were deceased when the present study was performed. Physical examinations and radiological examinations were conducted in the 4 affected patients (Table I), and all 4 patients presented with cutaneous syndactyly of the third and fourth fingers (Fig. 1B), without foot involvement. Blood samples were collected from 100 ethnically-matched unrelated normal controls (gender, 50 males, 50 females; age, 39.24 \pm 8.31 years) during June and October in 2013. They were recruited from the health examination center of the Third Xiangya Hospital of Central South University (Changsha, China) and examined by two professional surgeons according to the classification system described previously (9). All participants included in the present study provided written informed consent. The present study was performed in compliance with the Declaration of Helsinki, and was approved by the Institutional Review Board of the Third Xiangya Hospital of Central South University.

Exome capture. Genomic DNA (gDNA) was extracted from peripheral blood samples and used to extract the exome library using the phenol-chloroform extraction method (phenol, Beijing Solarbio Science \& Technology Co., Ltd., Beijing, China; chloroform, Tianjin Fuyu Fine Chemical Co., Ltd., Tianjin, China) as described previously (10). Genomic DNA from the proband (Fig. 1A: Generation IV, member 1) was randomly sheared using the Illumina Paired-End Sample Prep kit (Illumina, Inc., San Diego, CA, USA) and hybridized using SureSelect biotinylated RNA library and SureSelect hybridization buffers (Agilent Technologies, Inc., Santa Clara, CA, USA), as well as Optimized gDNA prep and library prep kits (Agilent Technologies, Inc.) to a concentration of $147 \mathrm{ng} / \mathrm{mg}$ at $45^{\circ} \mathrm{C}$ for enrichment; all experimental operations were performed according to the manufacturer's instructions for each kit. The enriched exome-targeting library was sequenced using the Illumina HiSeq 2000 platform (Illumina, Inc.) to generate $90-$ bp paired-end reads, as described previously (11). A mean exome coverage of $69.64 \mathrm{x}$ was obtained, thus allowing for the examination of selected regions at a sufficient depth to accurately match $99.42 \%$ of the targeted exome (12).

Exome sequencing and variant analysis. Human reference genomic sequences were obtained from the University of California Santa Cruz genome browser database (version hg19; build 37.1; http://genome.ucsc.edu/). Sequences obtained from the proband were aligned using the short oligonucleotide analysis package (SOAP) aligner software (soap2.21), and single nucleotide polymorphisms (SNPs) were identified using SOAPsnp software (version 1.03) (13). Small insertions or deletions in the coding sequence and splicing sites were detected as previously described $(11,12)$, and candidate SNPs were isolated. Variants were compared against several public databases including the Single Nucleotide Polymorphism database (dbSNP; build, 137; www.ncbi.nlm.nih.gov/projects/SNP/), the 1000 Genomes Project (http://www.1000genomes.org/), the International HapMap Project (http://hapmap.ncbi.nlm. nih.gov/) and the YanHuang project (http://yh.genomics.org. $\mathrm{cn} /$ ). Online tools including Sorting Intolerant from Tolerant (SIFT) prediction (http://sift.jcvi.org/), HumVar-trained Polymorphism Phenotyping version 2, (PolyPhen-2; http://genetics.bwh.harvard.edu/pph2/), and MutationTaster prediction (http://www.mutationtaster.org/) algorithms were used to estimate whether a variant may affect protein function $(14,15)$. The SIFT prediction scoring system ranges from 0 to 1 . Amino acid substitution is predicted as damaging (may have an important effect on the function of the protein) if the score is $\leq 0.05$, and tolerated (may have no effect on the function of the protein) if the score is $>0.05$. For PolyPhen-2, the predicted results include benign, possibly damaging and probably damaging. The PolyPhen-2 prediction scoring system ranges from 0 to 1 . The closer score is to 1 , the more influence the variant has on the function of the protein, and vice-versa. The predicted results of MutationTaster include disease causing (have an important effect on the function of the protein), disease causing automatic (have an important effect on the function of the protein in several public 
Table I. Clinical and genetic data of four patients from the same family harboring the HOXD13 c.917G>A (p.R306Q) mutation.

\begin{tabular}{|c|c|c|c|c|}
\hline \multirow{2}{*}{$\begin{array}{l}\text { Factor } \\
\text { Characteristic }\end{array}$} & \multicolumn{4}{|c|}{ Generation, member } \\
\hline & II, 1 & III, 2 & III, 4 & IV, 1 \\
\hline Sex & Male & Female & Female & Female \\
\hline Age (years) & 64 & 39 & 38 & 16 \\
\hline Genotype & Heterozygote & Heterozygote & Heterozygote & Heterozygote \\
\hline Syndactyly & Yes & Yes & Yes & Yes \\
\hline Affected fingers & 3rd and 4th & 3rd and 4th & 3rd and 4th & 3rd and 4th \\
\hline Bilateral affected & Yes & Yes & Yes & Yes \\
\hline Cutaneous fusion & Yes & Yes & Yes & Yes \\
\hline Bony fusion & No & No & No & No \\
\hline Toes affected & No & No & No & No \\
\hline Polydactyly & No & No & No & No \\
\hline Brachydactyly & No & No & No & No \\
\hline
\end{tabular}

HOXD13, homeobox D13 gene.

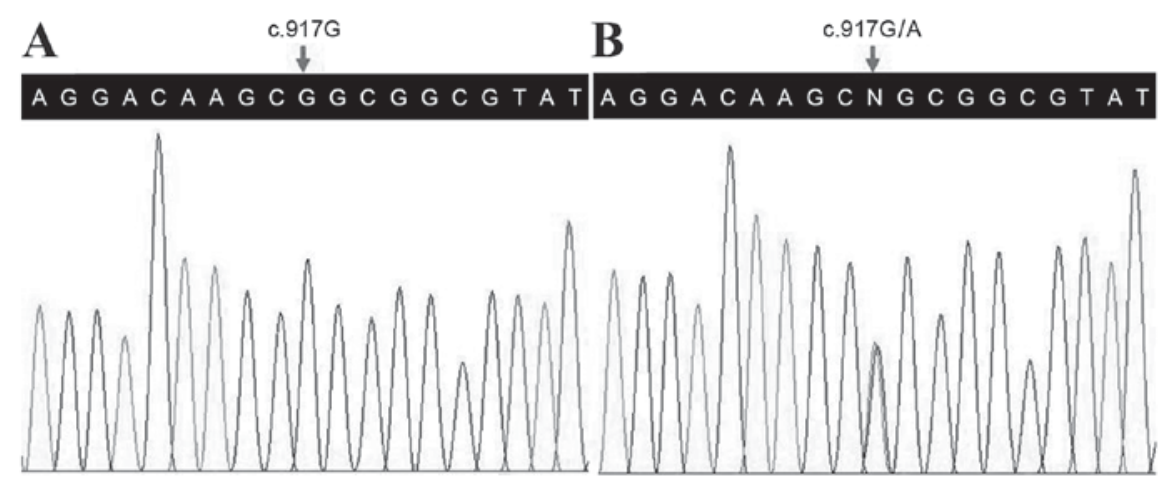

Figure 2. Sequencing analysis of the HOXD13 gene in (A) an unaffected family member (generation IV, member 2), and (B) the proband (generation IV, member 1). The proband was observed to harbor a heterozygous c.917G $>$ A (p.R306Q) mutation in HOXD13. HOXD13, homeobox D13 gene.

databases), polymorphism (may be a tolerant SNP) and polymorphism automatic (may be a tolerant SNP in several public databases). The probability of the prediction was measured by a scoring system ranging from 0 to 1 ; the closer the score was to 1 , the higher the probability of the prediction, and vice-versa $(14,15)$. Known mutations and genes responsible for syndactyly, nonsense mutations, read-through mutations and splicing mutations, or mutations predicted to be damaging were prioritized in identifying the pathogenic mutation. The National Center for Biotechnology Information-Basic Local Alignment Search Tool (NCBI-BLAST; http://blast.ncbi.nlm .nih.gov/Blast.cgi) was used to examine sequence conservation among different species.

Mutation validation. Polymerase chain reaction primers were designed using Primer 3 online primer design software (version 0.4.0; http://frodo.wi.mit.edu/primer3). Sanger sequencing was performed to validate the potential disease-associated variants using the ABI 3500 sequencer (Applied Biosystems; Thermo Fisher Scientific, Inc., Waltham, MA, USA) according to the standard procedures described previously $(16,17)$. The sequences of primers used to determine the causative variation in the HOXD13 gene were as follows:
Forward, 5'-GGGAGGAAGAAGAGAGTGCC-3' and reverse, 5'-TCTAAGCTGTCTGTGGCCAA-3'.

\section{Results}

Exome sequencing of the proband (Fig. 1A: Generation IV, member 1) in the syndactyly type I-c-affected family was first performed. A total of 5.92 billion bases of 90-bp paired-end read sequences for the patient were generated, and 106,958 genetic variants, including 14,830 non-synonymous alterations, were identified in the coding regions or the splicing sites. A prioritization scheme was applied to identify the pathogenic mutation, using similar methods described in a previous study (18). Given that the frequency of syndactyly type I is $0.03 \%$ (2), known variants identified in the dbSNP (build, 137), the 1000 Genomes Project, the International HapMap project and the YanHuang project with a minor allele frequency value of $>0.50 \%$ in each database, were excluded. Using the aforementioned criteria, the number of candidate genes were reduced by $>92.99 \%$. Variants not annotated in public databases were prioritized for further analysis.

Following validation by Sanger sequencing, a heterozygous mutation in the HOXD13 gene (c.917G>A, p.R306Q) was 


\begin{tabular}{lc} 
& \multicolumn{1}{c}{ p.R306 } \\
Homo sapiens & INKFINKDKRRRISAATNL \\
Pan troglodytes & INKFINKDKRRRISAATNL \\
Callithrix jacchus & LNKFINKDKRRRISAATNL \\
Sus scrofa & INKFINKDKRRRISAATNL \\
Bos taurus & VNKFINKDKRRRISAATNL \\
Equus przewalskii & INKFINKDKRRRISAATNL \\
Ovis aries & VNKFINKDKRRRISAATNL \\
Felis catus & INKFINKDKRRRISAATNL \\
Oryctolagus cuniculus & INKFINKDKRRRISAATNL \\
Mus musculus & INKFINKDKRRRISAATNL \\
Rattus norvegicus & INKFINKDKRRRISAATNL \\
Gallus gallus & INKFINKDKRRRISAATNL \\
Lipotes vexillifer & INKFINKDKRRRISAATNL
\end{tabular}

Figure 3. Conservation analysis of the p.R306 amino acid residue in the homeobox-D13 sequence among 13 different species.

observed in the proband (Fig. 2), however, no other variants in the known disease-causing genes for syndactyly were identified by exome sequencing. The mutation co-segregated among the affected family members, and was absent in the unaffected family members, as well as in the 100 normal controls. This suggests that the c. $917 \mathrm{G}>$ A mutation in $H O X D 13$ may have been the pathogenic mutation. In further support of this notion, this mutation was also absent in an in-house database from the BGI-Shenzhen (Shenzhen, China) with 2,375 ethnically-matched controls. The results indicate that the c.917G >A (p.R306Q) variant of the HOXD13 gene may be the disease-associated mutation in this family. The c.917G $>A$ mutation is located in exon 2 , which encodes the homeodomain in the C-terminal region (a highly-conserved DNA binding motif) that is responsible for binding to consensus DNA sequences (19). The mutation alters the amino acid sequence at position 306 from Arg to Gln at residue 31 of this homeodomain. This position was observed to be highly conserved among different species (Fig. 3), indicating its structural and functional importance. This mutation was predicted to be disease-associated and affect protein function using the MutationTaster and SIFT prediction algorithms, and potentially damaging using the PolyPhen-2 algorithm (score, 1.00).

\section{Discussion}

Syndactyly displays marked genetic heterogeneity and high clinical variability, and is characterized by limb malformation (1). Syndactyly type I-c is the rarest type among the four subtypes of syndactyly type I (I-a, I-b, I-c and I-d), which is the most common form of non-syndromic syndactyly (2). Two mutations (p.R306Q and p.R306 G) in the HOXD13 homeodomain have been identified in two independent Chinese families diagnosed with syndactyly type I-c in a previous study (20). However, inconsistencies between the clinical phenotypes and diagnostic criteria in selected patients from the two families were observed, including a mild synpolydactyly phenotype, involvement of the feet, clinodactyly and fusion of the second and third fingers, which questions the accuracy of the clinical diagnosis (20). The present study included a Chinese Han family consisting of members diagnosed with typical syndactyly type I-c that presented with bilateral cutaneous webbing of the third and fourth fingers, normal toes, and an absence of additional abnormalities. The results, together with those obtained from the different prediction programs, identified the $H O X D 13$ c.917G $>$ A (p.R306Q) variant as the potential pathogenic mutation for this family. Given that $\mathrm{G}>\mathrm{A}$ or $\mathrm{C}>\mathrm{T}$ transitions within $\mathrm{CpG}$ dinucleotides are reportedly responsible for one-third of single base-pair mutations in gene coding regions in human genetic diseases (21), the c.917G > A mutation may be caused by cytosine methylation followed by deamination to thymine $(\mathrm{C}>\mathrm{T})$ on the complementary strand.

To the best of our knowledge, this is the first study to present a family diagnosed with syndactyly type I-c, with affected members harboring the HOXD13 p.R306Q mutation with complete penetrance. The two independent Chinese families with syndactyly reported previously (20), may have been diagnosed with unknown complex types of non-syndromic syndactyly or syndactyly type I-c variant types with incomplete penetrance and atypical phenotypes. As two different amino acid substitutions from different families have been identified at position 306 , this position may be a mutation hotspot. In addition, in a previous study, common mutation screening was unable to exclude the genes involved in finger/toe development, which was excluded by exome sequencing used in the present study (20).

The HOXD13 gene is a member of the HOX gene family, and encodes a transcription factor that serves a crucial role in limb development (22). HOXD13 is composed of 2 exons and encodes a 343-amino acid polypeptide sequence (23). At least 36 different $H O X D 13$ mutations, including 17 missense/nonsense mutations, 8 repeats, 6 small deletions, 2 splicing mutations, a small insertion, a gross deletion, as well as a gross insertion/duplication mutation, have been described in the Human Gene Mutation Database (http://www.hgmd.cf.ac.uk/ac/index. php). The majority of these mutations are observed in patients with limb malformations, including syndactyly, synpolydactyly and brachydactyly (24). Mouse models with different genetic deficiencies in the HOXD13 gene have been described previously in the literature. The majority of these mouse models mimic some aspects of human synpolydactyly phenotypes, further supporting the importance of the HOXD13 gene in limb development (25-27).

In conclusion, the results of the present study indicate that the c.917G >A (p.R306Q) mutation in the HOXD13 gene may be the genetic basis of syndactyly type I-c in a single Chinese family, which increases the phenotypic spectrum of the HOXD13 gene. Whole exome sequencing provides a cost-effective and expedited approach for identifying disease-associated genes, and excludes the effects of background genes in disorders displaying high clinical heterogeneity. The results of the present study may provide novel insights into the genetic diagnosis of syndactyly, and may have implications for genetic counseling and the clinical management of this condition.

\section{Acknowledgements}

The authors would like to thank the participating members and investigators for their cooperation and efforts in 
collecting clinical and genetic information and DNA specimens. The present study was supported by grants from the National Key Research and Development Program of China (grant no. 2016YFC1306604), the National Natural Science Foundation of China (grant no. 81670216), the Natural Science Foundation of Hunan Province (grant nos. 2015JJ4088 and 2016JJ2166), the Grant for the Foster Key Subject of the Third Xiangya Hospital Clinical Laboratory Diagnostics (H.D.), Zhishan Lead Project of the Third Xiangya Hospital (grant no. 20150301) and the Fundamental Research Funds for the Central Universities of Central South University (grant no. 2015zzts318).

\section{References}

1. Malik S: Syndactyly: Phenotypes, genetics and current classification. Eur J Hum Genet 20: 817-824, 2012.

2. Malik S, Schott J, Ali SW, Oeffner F, Amin-ud-Din M, Ahmad W, Grzeschik KH and Koch MC: Evidence for clinical and genetic heterogeneity of syndactyly type I: The phenotype of second and third toe syndactyly maps to chromosome 3p21.31. Eur J Hum Genet 13: 1268-1274, 2005.

3. Jordan D, Hindocha S, Dhital M, Saleh M and Khan W: The epidemiology, genetics and future management of syndactyly. Open Orthop J 6: 14-27, 2012.

4. Sobreira NL, Cernach MC, Brunoni D and Perez AB: Complex toe syndactyly with characteristic facial phenotype: A new syndrome? Am J Med Genet A 146A: 1725-1728, 2008.

5. Fujii S, Yabe K, Kimura Y, Ito Y, Rokukawa M, Furukawa M, Ito K, Matsuura M and Kiguchi M: Syndactyly lethal: New mutation with multiple malformations occurring in Sprague Dawley rats. Congenit Anom (Kyoto) 49: 262-268, 2009.

6. Jamsheer A, Zemojtel T, Kolanczyk M, Stricker S, Hecht J Krawitz P, Doelken SC, Glazar R, Socha M and Mundlos S: Whole exome sequencing identifies FGF16 nonsense mutations as the cause of X-linked recessive metacarpal $4 / 5$ fusion. J Med Genet 50: 579-584, 2013.

7. Zhou X, Zheng C, He B, Zhu Z, Li P, He X, Zhu S, Yang C, Lao Z, Zhu Q and Liu X: A novel mutation outside homeodomain of HOXD13 causes synpolydactyly in a Chinese family. Bone 57: 237-241, 2013

8. Zhao X, Sun M, Zhao J, Leyva JA, Zhu H, Yang W, Zeng X, Ao Y, Liu Q, Liu G, et al: Mutations in HOXD13 underlie syndactyly type V and a novel brachydactyly-syndactyly syndrome. Am J Hum Genet 80: 361-371, 2007.

9. Deng $\mathrm{H}$ and Tan T: Advances in the molecular genetics of non-syndromic syndactyly. Curr Genomics 16: 183-193, 2015.

10. Guo Y, Yuan L, Yi J, Xiao J, Xu H, Lv H, Xiong W, Zheng W, Guan L, Zhang J, et al: Identification of a GJA3 mutation in a Chinese family with congenital nuclear cataract using exome sequencing. Indian J Biochem Biophys 50: 253-258, 2013.
11. Wang JL, Cao L, Li XH, Hu ZM, Li JD, Zhang JG, Liang Y, San-A, Li N, Chen SQ, et al: Identification of PRRT2 as the causative gene of paroxysmal kinesigenic dyskinesias. Brain 134: 3490-3498, 2011

12. Shi Y, Li Y, Zhang D, Zhang H, Li Y, Lu F, Liu X, He F, Gong B, Cai L, et al: Exome sequencing identifies ZNF644 mutations in high myopia. PLoS Genet 7: e1002084, 2011.

13. Li R, Li Y, Kristiansen K and Wang J: SOAP: Short oligonucleotide alignment program. Bioinformatics 24: 713-714, 2008.

14. $\mathrm{Hu} \mathrm{J}$ and Ng PC: SIFT Indel: Predictions for the functional effects of amino acid insertions/deletions in proteins. PLoS One 8: e77940, 2013.

15. Adzhubei I, Jordan DM and Sunyaev SR: Predicting functional effect of human missense mutations using PolyPhen-2. Curr Protoc Hum Genet Chapter 7: Unit 7.20, 2013.

16. Lei J, Deng X, Zhang J, Su L, Xu H, Liang H, Huang X, Song Z and Deng H: Mutation screening of the HDC gene in Chinese Han patients with Tourette syndrome. Am J Med Genet B Neuropsychiatr Genet 159B: 72-76, 2012.

17. Yuan L, Song Z, Xu H, Gu S, Zhu A, Gong L, Zhao Y and Deng H: EIF4G1 Ala502Val and Arg1205His variants in Chinese patients with Parkinson disease. Neurosci Lett 543: 69-71, 2013.

18. Guo Y, Yuan J, Liang H, Xiao J, Xu H, Yuan L, Gao K, Wu B, Tang Y, Li X and Deng H: Identification of a novel COL4A5 mutation in a Chinese family with X-linked Alport syndrome using exome sequencing. Mol Biol Rep 41: 3631-3635, 2014.

19. Brison N, Tylzanowski $P$ and Debeer P: Limb skeletal malformations-what the HOX is going on? Eur J Med Genet 55: 1-7, 2012.

20. Dai L, Liu D, Song M, Xu X, Xiong G, Yang K, Zhang K, Meng $\mathrm{H}$, Guo H and Bai Y: Mutations in the homeodomain of HOXD13 cause syndactyly type 1-c in two Chinese families. PLoS One 9: e96192, 2014.

21. Cooper DN and Youssoufian $\mathrm{H}$ : The $\mathrm{CpG}$ dinucleotide and human genetic disease. Hum Genet 78: 151-155, 1988.

22. Kurban M, Wajid M, Petukhova L, Shimomura Y and Christiano AM: A nonsense mutation in the HOXD13 gene underlies synpolydactyly with incomplete penetrance. J Hum Genet 56: 701-706, 2011.

23. Akarsu AN, Stoilov I, Yilmaz E, Sayli BS and Sarfarazi M: Genomic structure of HOXD13 gene: A nine polyalanine duplication causes synpolydactyly in two unrelated families. Hum Mol Genet 5: 945-952, 1996.

24. Jamsheer A, Sowinska A, Kaczmarek L and Latos-Bielenska A: Isolated brachydactyly type E caused by a HOXD13 nonsense mutation: A case report. BMC Med Genet 13: 4, 2012.

25. Bruneau S, Johnson KR, Yamamoto M, Kuroiwa A and Duboule D: The mouse Hoxd13(spdh) mutation, a polyalanine expansion similar to human type II synpolydactyly (SPD), disrupts the function but not the expression of other Hoxd genes. Dev Biol 237: 345-353, 2001.

26. Zakany J and Duboule D: Synpolydactyly in mice with a targeted deficiency in the HoxD complex. Nature 384: 69-71, 1996.

27. Dollé P, Dierich A, LeMeur M, Schimmang T, Schuhbaur B, Chambon P and Duboule D: Disruption of the Hoxd-13 gene induces localized heterochrony leading to mice with neotenic limbs. Cell 75: 431-441, 1993. 